\title{
Intuitionistic fuzzy l-convergent Fibonacci difference sequence spaces
}

\author{
Vakeel A. Khan ${ }^{1 *}$, Emrah E. Kara², Henna Altaf ${ }^{1}$, Nazneen Khan ${ }^{3}$ and Mobeen Ahmad
}

\section{"Correspondence:}

vakhanmaths@gmail.com

'Department of Mathematics,

Aligarh Muslim University, Aligarh,

India

Full list of author information is

available at the end of the article

\begin{abstract}
Fibonacci difference matrix was defined by Kara in his paper (Kara in J. Inequal. Appl. 2013:38 2013). Recently, Khan et al. (Adv. Differ. Equ. 2018:199, 2018) using the Fibonacci difference matrix $\hat{F}$ and ideal convergence defined the notion of $c_{0}^{\prime}(\hat{F}), c^{\prime}(\hat{F})$ and $I_{\infty}^{\prime}(\hat{F})$. In this paper, we give the ideal convergence of Fibonacci difference sequence space in intuitionistic fuzzy normed space with respect to fuzzy norm $(\mu, v)$. Moreover, we investigate some basic properties of the said spaces such as linearity, hausdorffness.
\end{abstract}

Keywords: Difference sequence space; Fibonacci numbers; Fibonacci difference matrix; Intuitionistic fuzzy normed space; I-convergence

\section{Introduction and preliminaries}

Let $\omega, c, c_{0}, l_{\infty}$ denote sequence space, convergent, null and bounded sequences respectively, with norm $\|x\|_{\infty}=\sup _{k \in \mathbb{N}}\left|x_{k}\right|$. The idea of difference sequence spaces was defined by Kizmaz as follows:

$$
\lambda(\Delta)=\left\{x=\left(x_{n}\right) \in \omega:\left(x_{n}-x_{n+1}\right) \in \lambda\right\}, \quad \text { for } \lambda \in\left\{l_{\infty}, c, c_{0}\right\} .
$$

Recently, many authors have made a new approach to construct sequence spaces using matrix domain $[2,3,8,10]$. Lately, Kara [9] has investigated difference sequence space.

$$
l_{\infty}(\hat{F})=\left\{x=\left(x_{n}\right) \in \omega: \sup _{n \in \mathbb{N}}\left|\frac{f_{n}}{f_{n+1}} x_{n}-\frac{f_{n+1}}{f_{n}} x_{n-1}\right|<\infty\right\}
$$

which is derived from the Fibonacci difference matrix $\hat{F}=\left(\hat{f_{n k}}\right)$ as follows:

$$
\hat{f_{n k}}= \begin{cases}-\frac{f_{n+1}}{f_{n}} & k=n-1, \\ \frac{f_{n}}{f_{n+1}} & k=n, \\ 0 & 0 \leq k<n-1 \text { or } k>n,\end{cases}
$$

where $\left(f_{n}\right), n \in \mathbb{N}$ is the sequence of Fibonacci numbers given by the linear recurrence relation as $f_{0}=1=f_{1}$ and $f_{n-1}+f_{n-2}=f_{n}$ for $n \geq 2$. Quite recently, Khan et al. [13] defined the notion of $I$-convergent Fibonacci difference sequence spaces as $c_{0}^{I}(\hat{F}), c^{I}(\hat{F})$ and $l_{\infty}^{I}(\hat{F})$.

(c) The Author(s) 2019. This article is distributed under the terms of the Creative Commons Attribution 4.0 International License (http://creativecommons.org/licenses/by/4.0/), which permits unrestricted use, distribution, and reproduction in any medium, provided you give appropriate credit to the original author(s) and the source, provide a link to the Creative Commons license, and indicate if changes were made. 
Fibonacci numbers have various applications in the fields of arts, science and architecture. For further details, refer to $[4,11]$.

Fuzzy logic was first introduced by Zadeh in 1965 [24] and it found its applications in various fields like control theory, artificial intelligence, robotics. Later on many authors $[7,16]$ investigated fuzzy topology to define fuzzy metric space. As a generalisation of fuzzy sets, Atanassov [1] defined the view of intuitionistic fuzzy sets. Intuitionistic fuzzy normed space [18] and 2-normed space [17] are the recent studies in fuzzy theory.

Kostyrko et al. [15] in 1999 generalised the idea of statistical convergence [6, 21] to ideal convergence. Further this idea was investigated by Salat, Tripathy and Ziman [19, 20], Tripathy and Hazarika [22, 23] and many others.

We recall certain definitions which will be useful in this paper.

Definition 1.1 ([21]) A sequence $x=\left(x_{n}\right) \in \omega$ is statistically convergent to $\xi \in \mathbb{R}$ if for every $\epsilon>0$ the set $\left\{n \in \mathbb{N}:\left|x_{n}-\xi\right| \geq \epsilon\right\}$ has asymptotic density zero. We write $s t-\lim x=\xi$. If $\xi=0$, then $x=\left(x_{n}\right)$ is called st-null.

Definition 1.2 A sequence $x=\left(x_{n}\right) \in \omega$ is called statistically Cauchy sequence if, for every $\epsilon>0, \exists$ a number $N=N(\epsilon)$ such that

$$
\lim _{n} \frac{1}{n}\left|\left\{j \leq n:\left|x_{j}-x_{N}\right| \geq \epsilon\right\}\right|=0 .
$$

Definition 1.3 ([14]) An ideal means a family of sets $I \subset P(X)$ satisfying the following conditions:

(i) $\phi \in I$,

(ii) $C \cup D \in I$ for all $C, D \in I$,

(iii) for each $C \in I$ and $D \subset C$, we have $D \in I$.

An ideal is said to be non-trivial if $I \neq 2^{X}$ and admissible if $\{\{x\}: x \in X\} \subset I$.

Definition 1.4 ([13]) A family of sets $\mathcal{F} \subset P(X)$ is called filter if and only if it satisfies the following conditions:

(i) $\phi \notin \mathcal{F}$,

(ii) $C, D \in \mathcal{F} \Rightarrow C \cap D \in \mathcal{F}$,

(iii) for each $C \in \mathcal{F}$ with $C \subset D$, we have $D \in \mathcal{F}$.

Definition 1.5 ([15]) A sequence $x=\left(x_{n}\right)$ is called $I$-convergent to $\xi \in \mathbb{R}$ if, for every $\epsilon>0$, the set $\left\{n \in \mathbb{N}:\left|x_{n}-\xi\right| \geq \epsilon\right\} \in I$. We write $I-\lim x=\xi$. If $\xi=0$, then $x=\left(x_{n}\right)$ is said to be $I$-null.

Definition 1.6 ([13]) A sequence $x=\left(x_{n}\right)$ is said to be $I$-Cauchy if for every $\epsilon>0 \exists$ a number $N=N(\epsilon)$ such that the set $\left\{n \in \mathbb{N}:\left|x_{n}-x_{N}\right| \geq \epsilon\right\} \in \mathcal{I}$.

Definition 1.7 ([14]) A sequence $x=\left(x_{k}\right)$ is convergent to $\xi$ with respect to the intuitionistic fuzzy norm $(\mu, v)$ if for every $\epsilon, t>0 \exists N \in \mathbb{N}$ with $\mu\left(x_{k}-\xi, t\right)>1-\epsilon$ and $\nu\left(x_{k}-\xi, t\right)<\epsilon$ for all $k \geq N$. We write $(\mu, v)-\lim x=\xi$.

Definition 1.8 ([5]) Consider intuitionistic fuzzy normed space (IFNS) $(X, \mu, \nu, *, \diamond)$. A sequence $x=\left(x_{k}\right)$ is said to be Cauchy sequence with respect to norm $(\mu, v)$ if, for each $\epsilon>0, t>0$, there exists $N \in \mathbb{N}$ such that $\mu\left(x_{k}-x_{l}, t\right)>1-\epsilon$ and $v\left(x_{k}-x_{l}\right)<\epsilon$ for all $k, l \geq N$. 
Definition 1.9 ([12]) Let $(X, \mu, \nu, *, \diamond)$ be IFNS. A sequence $x=\left(x_{k}\right) \in \omega$ is called $I$ convergent to $\xi$ with respect to the intuitionistic norm $(\mu, \nu)$ if for every $\epsilon, t>0$ if the set $\left\{k \in \mathbb{N}: \mu\left(x_{k}-\xi, t\right) \leq 1-\epsilon\right.$ or $\left.v\left(x_{k}-\xi, t\right) \geq \epsilon\right\} \in I$. We write $I^{(\mu, v)}-\lim x=\xi$.

Definition 1.10 ([13]) A sequence $x=\left(x_{k}\right) \in \omega$ is said to be Fibonacci $I$-convergent to $\xi \in \mathbb{R}$ if, for every $\epsilon>0$, the set $\left\{k \in \mathbb{N}:\left|\hat{F}_{k}(x)-\xi\right| \geq \epsilon\right\} \in I$, where $I$ is an admissible ideal.

Definition 1.11 ([13]) Consider an admissible ideal $I$. Sequence $x=\left(x_{k}\right) \in \omega$ is Fibonacci $I$-Cauchy if, for every $\epsilon>0, \exists N=N(\epsilon)$ such that $\left\{k \in \mathbb{N}:\left|\hat{F}_{k}(x)-\hat{F}_{N}(x)\right| \geq \epsilon\right\} \in I$.

\section{Intuitionistic fuzzy I-convergent Fibonacci difference sequence spaces}

In the following section, we introduce a new type of sequence spaces whose $\hat{F}$ transform is $I$-convergent with respect to the intuitionistic norm $(\mu, v)$. Further we prove certain properties of these spaces such as hausdorfness, first countability. Throughout this paper, $I$ is an admissible ideal. We define

$$
\begin{aligned}
& S_{0(\mu, v)}^{I}(\hat{F})=\left\{x=\left(x_{k}\right) \in l_{\infty}:\left\{k \in \mathbb{N}: \mu\left(\hat{F}_{k}(x), t\right) \leq 1-\epsilon \text { or } v\left(\hat{F}_{k}(x), t\right) \geq \epsilon\right\} \in I\right\}, \\
& S_{(\mu, \nu)}^{I}(\hat{F})=\left\{x=\left(x_{k}\right) \in l_{\infty}:\left\{k \in \mathbb{N}: \mu\left(\hat{F}_{k}(x)-l, t\right) \leq 1-\epsilon \text { or } v\left(\hat{F}_{k}(x)-l, t\right) \geq \epsilon\right\} \in I\right\} .
\end{aligned}
$$

We introduce an open ball with centre $x$ and radius $r$ with respect to $t$ as follows:

$$
\begin{aligned}
B_{x}(r, t)(\hat{F})= & \left\{y=\left(y_{k}\right) \in l_{\infty}:\right. \\
& \left\{k \in \mathbb{N}: \mu\left(\hat{F}_{k}(x)-\hat{F}_{k}(y), t\right)>1-r \text { and } v\left(\hat{F}_{k}(x)-\hat{F}_{k}(y), t\right)<r\right\} .
\end{aligned}
$$

\section{Remark 2.1}

(i) For $p_{1}, p_{2} \in(0,1)$ such that $p_{1}>p_{2}$, there exist $p_{3}, p_{4} \in(0,1)$ with $p_{1} * p_{3} \geq p_{2}$ and $p_{1} \geq p_{4} \diamond p_{2}$.

(ii) For $p_{5} \in(0,1)$, there exist $p_{6}, p_{7} \in(0,1)$ such that $p_{6} * p_{6} \geq p_{5}$ and $p_{7} \diamond p_{7} \leq p_{5}$.

Theorem 2.1 The spaces $S_{0(\mu, v)}^{I}(\hat{F})$ and $S_{(\mu, v)}^{I}(\hat{F})$ are vector spaces over $\mathbb{R}$.

Proof Let us show the result for $S_{(\mu, v)}^{I}(\hat{F})$ and the proof for another space will follow on the similar lines. Let $x=\left(x_{k}\right)$ and $y=\left(y_{k}\right) \in S_{(\mu, \nu)}^{I}(\hat{F})$. Then by definition there exist $\xi_{1}$ and $\xi_{2}$, and for every $\epsilon, t>0$, we have

$$
\begin{aligned}
& A=\left\{k \in \mathbb{N}: \mu\left(\hat{F}_{k}(x)-\xi_{1}, \frac{t}{2|\alpha|}\right) \leq 1-\epsilon \text { or } \nu\left(\hat{F}_{k}(x)-\xi_{1}, \frac{t}{2|\alpha|}\right) \geq \epsilon\right\} \epsilon, \\
& B=\left\{k \in \mathbb{N}: \mu\left(\hat{F}_{k}(y)-\xi_{2}, \frac{t}{2|\beta|}\right) \leq 1-\epsilon \text { or } \nu\left(\hat{F}_{k}(y)-\xi_{2}, \frac{t}{2|\beta|}\right) \geq \epsilon\right\} \in I,
\end{aligned}
$$

where $\alpha$ and $\beta$ are scalars.

$$
\begin{aligned}
& A^{c}=\left\{k \in \mathbb{N}: \mu\left(\hat{F}_{k}(x)-\xi_{1}, \frac{t}{2|\alpha|}\right)>1-\epsilon \text { or } v\left(\hat{F}_{k}(x)-\xi_{1}, \frac{t}{2|\alpha|}\right)<\epsilon\right\} \in \mathcal{F}(I), \\
& B^{c}=\left\{k \in \mathbb{N}: \mu\left(\hat{F}_{k}(y)-\xi_{2}, \frac{t}{2|\beta|}\right)>1-\epsilon \text { or } v\left(\hat{F}_{k}(y)-\xi_{2}, \frac{t}{2|\beta|}\right)<\epsilon\right\} \in \mathcal{F}(I) .
\end{aligned}
$$


Define $E=A \cup B$ so that $E \in I$. Thus $E^{c} \in \mathcal{F}(I)$ and therefore is non-empty. We will show

$$
\begin{gathered}
E^{c} \subset\left\{k \in \mathbb{N}: \mu\left(\alpha \hat{F}_{k}(x)+\beta \hat{F}_{k}(y)-\left(\alpha \xi_{1}+\beta \xi_{2}\right), t\right)>1-\epsilon\right. \text { or } \\
\left.v\left(\alpha \hat{F}_{k}(x)+\beta \hat{F}_{k}(y)-\left(\alpha \xi_{1}+\beta \xi_{2}\right), t\right)<\epsilon\right\} .
\end{gathered}
$$

Let $n \in E^{c}$. Then

$$
\begin{aligned}
& \mu\left(\hat{F}_{n}(x)-\xi_{1}, \frac{t}{2|\alpha|}\right)>1-\epsilon \text { or } v\left(\hat{F}_{n}(x)-\xi_{1}, \frac{t}{2|\alpha|}\right)<\epsilon, \\
& \mu\left(\hat{F}_{n}(y)-\xi_{2}, \frac{t}{2|\beta|}\right)>1-\epsilon \text { or } v\left(\hat{F}_{n}(y)-\xi_{2}, \frac{t}{2|\beta|}\right)<\epsilon
\end{aligned}
$$

Consider

$$
\begin{aligned}
& \mu\left(\alpha \hat{F}_{n}(x)+\beta \hat{F}_{n}(x)-\left(\alpha \xi_{1}+\beta \xi_{2}\right), t\right) \\
& \quad \geq \mu\left(\alpha \hat{F}_{n}(x)-\alpha \xi_{1}, \frac{t}{2}\right) * \mu\left(\beta \hat{F}_{n}(y)-\beta \xi_{2}, \frac{t}{2}\right) \\
& \quad=\mu\left(\hat{F}_{n}(x)-\xi_{1}, \frac{t}{2|\alpha|}\right) * \mu\left(\hat{F}_{n}(y)-\xi_{2}, \frac{t}{|\beta|}\right) \\
& \quad>(1-\epsilon) *(1-\epsilon)=1-\epsilon
\end{aligned}
$$

and

$$
\begin{aligned}
& v\left(\alpha \hat{F}_{n}(y)+\beta \hat{F}_{n}(y)-\left(\alpha \xi_{1}+\beta \xi_{2}\right)\right) \\
& \quad \leq v\left(\alpha \hat{F}_{n}(x)-\alpha \xi_{1}, \frac{t}{2}\right) \diamond v\left(\beta \hat{F}_{n}(y)-\beta \xi_{2}, \frac{t}{2}\right) \\
& \quad=v\left(\hat{F}_{n}(x)-\xi_{1}, \frac{t}{2|\alpha|}\right) \diamond v\left(\hat{F}_{n}(y)-\xi_{2}, \frac{t}{2|\beta|}\right) \\
& \quad<\epsilon \diamond \epsilon=\epsilon .
\end{aligned}
$$

Thus $E^{c} \subset\left\{k \in \mathbb{N}: \mu\left(\alpha \hat{F}_{k}(x)+\beta \hat{F}_{k}(y)-\left(\alpha \xi_{1}+\beta \xi_{2}\right), t\right)>1-\epsilon\right.$ or $v\left(\alpha \hat{F}_{k}(x)+\beta \hat{F}_{k}(y)-\left(\alpha \xi_{1}+\right.\right.$ $\left.\left.\left.\beta \xi_{2}\right), t\right)<\epsilon\right\} . E^{c} \in \mathcal{F}(I)$, therefore by definition of filter, the set on the right-hand side of the above equation belongs to $\mathcal{F}(I)$ so that its complement belongs to $I$. This implies $(\alpha x+$ $\beta y) \in S_{(\mu, v)}^{I}(\hat{F})$. Hence $S_{(\mu, v)}^{I}(\hat{F})$ is a vector space over $\mathbb{R}$.

Theorem 2.2 Every open ball $B_{x}(r, t)(\hat{F})$ is an open set in $S_{(\mu, v)}^{I}(\hat{F})$.

Proof We have defined open ball as follows:

$$
\begin{aligned}
B_{x}(r, t)(\hat{F})= & \left\{y=\left(y_{k}\right) \in l_{\infty}:\right. \\
& \left\{k \in \mathbb{N}: \mu\left(\hat{F}_{k}(x)-\hat{F}_{k}(y), t\right)>1-r \text { and } v\left(\hat{F}_{k}(x)-\hat{F}_{k}(y), t\right)<r\right\} .
\end{aligned}
$$

Let $z=\left(z_{k}\right) \in B_{x}(r, t)(\hat{F})$ so that $\mu\left(\hat{F}_{k}(x)-\hat{F}_{k}(z), t\right)>1-r$ and $v\left(\hat{F}_{k}(x)-\hat{F}_{k}(z), t\right)<r$. Then there exists $t_{0} \in(0, t)$ with $\mu\left(\hat{F}_{k}(x)-\hat{F}_{k}(z), t_{0}\right)>1-r$ and $v\left(\hat{F}_{k}(x)-\hat{F}_{k}(z), t_{0}\right)<r$. Put $p_{0}=$ 
$\mu\left(\hat{F}_{k}(x)-\hat{F}_{k}(z), t_{0}\right)$, so we have $p_{0}>1-r$, there exists $s \in(0,1)$ such that $p_{0}>1-s>1-r$. Using Remark 2.1(i), given $p_{0}>1-s$, we can find $p_{1}, p_{2} \in(0,1)$ with $p_{0} * p_{1}>1-s$ and $\left(1-p_{0}\right) \diamond\left(1-p_{2}\right)<s$. Put $p_{3}=\max \left(p_{1}, p_{2}\right)$. We will prove $B_{z}\left(1-p_{3}, t-t_{0}\right)(\hat{F}) \subset B_{x}(r, t)(\hat{F})$. Let $w=\left(w_{k}\right) \in B_{z}\left(1-p_{3}, t-t_{0}\right)(\hat{F})$. Hence

$$
\begin{aligned}
& \mu\left(\hat{F}_{k}(x)-\hat{F}_{k}(w), t\right) \geq \mu\left(\hat{F}_{k}(x)-\hat{F}_{k}(z), t_{0}\right) * \mu\left(\hat{F}_{k}(z)-\hat{F}_{k}(w), t-t_{0}\right) \\
& \quad>\left(p_{0} * p_{3}\right) \geq\left(p_{0} * p_{1}\right)>1-s>1-r,
\end{aligned}
$$

and

$$
\begin{aligned}
& v\left(\hat{F}_{k}(x)-\hat{F}_{k}(w), t\right) \leq v\left(\hat{F}_{k}(x)-\hat{F}_{k}(z), t_{0}\right) \diamond v\left(\hat{F}_{k}(z)-\hat{F}_{k}(w), t-t_{0}\right) \\
& \left.\quad<\left(1-p_{0}\right)\right) \diamond\left(1-p_{3}\right) \leq\left(1-p_{0}\right) \diamond\left(1-p_{2}\right)<r .
\end{aligned}
$$

Hence $w \in B_{x}(r, t)(\hat{F})$ and therefore $B_{z}\left(1-p_{3}, t-t_{0}\right)(\hat{F}) \subset B_{x}(r, t)(\hat{F})$.

Remark 2.2 Let $S_{(\mu, \nu)}^{I}\left(\hat{F}\right.$ be IFNS. Define $\tau_{(\mu, \nu)}^{I}(\hat{F})=\left\{A \subset S_{(\mu, \nu)}^{I}(\hat{F})\right.$ : for given $x \in A$, we can find $t>0$ and $0<r<1$ such that $\left.B_{x}(r, t)(\hat{F}) \subset A\right\}$. Then $\tau_{(\mu, v)}^{I}(\hat{F})$ is a topology on $S_{(\mu, \nu)}^{I}(\hat{F})$.

Remark 2.3 Since $\left\{B_{x}\left(\frac{1}{n}, \frac{1}{n}\right)(\hat{F}): n \in \mathbb{N}\right\}$ is a local base at $x$, the topology $\tau_{(\mu, \nu)}^{I}(\hat{F})$ is first countable.

Theorem 2.3 The spaces $S_{(\mu, v)}^{I}(\hat{F})$ and $S_{0(\mu, v)}^{I}(\hat{F})$ are Hausdorff.

Proof Let $x, y \in S_{(\mu, \nu)}^{I}(\hat{F})$ with $x$ and $y$ to be different. Then $0<\mu\left(\hat{F}_{k}(x)-\hat{F}(y), t\right)<1$ and $0<v\left(\hat{F}(x)-\hat{F}_{k}(y), t\right)<1$. Put $\mu\left(\hat{F}_{k}(x)-\hat{F}_{k}(y), t\right)=p_{1}$ and $v\left(\hat{F}_{k}(x)-\hat{F} k(y), t\right)=p_{2}$ and $r=$ $\max \left(p_{1}, 1-p_{2}\right)$. Using Remark $(2.1(i i))$ for $p_{0} \in(r, 1)$, we can find $p_{3}, p_{4} \in(0,1)$ such that $p_{3} * p_{3} \geq p_{0}$ and $\left(1-p_{4}\right) \diamond\left(1-p_{4}\right) \leq 1-p_{0}$. Put $p_{5}=\max \left(p_{3}, p_{4}\right)$. Clearly $B_{x}\left(1-p_{5}, \frac{t}{2}\right)(\hat{F}) \cap$ $B_{y}\left(1-p_{5}, \frac{t}{2}\right)(\hat{F})=\phi$. Let on the contrary $z \in B_{x}\left(1-p_{5}, \frac{t}{2}\right)(\hat{F}) \cap B_{y}\left(1-p_{5}, \frac{t}{2}\right)(\hat{F})$. Then we have

$$
\begin{aligned}
p_{1} & =\mu\left(\hat{F}_{k}(x)-\hat{F}_{k}(y), t\right) \geq \mu\left(\hat{F}_{k}(x)-\hat{F}_{k}(z), \frac{t}{2}\right) * \mu\left(\hat{F}_{k}(z)-\hat{F}_{k}(y), \frac{t}{2}\right) \\
& \geq p_{5} * p_{5} \geq p_{3} * p_{3}>p_{0}>p_{1}
\end{aligned}
$$

and

$$
\begin{aligned}
p_{2} & =v\left(\hat{F}_{k}(x)-\hat{F}_{y}, t\right) \leq v\left(\hat{F}_{k}(x)-\hat{F}_{k}(z), \frac{t}{2}\right) \diamond v\left(\hat{F}_{k}(z)-\hat{F}_{k}(y), \frac{t}{2}\right) \\
& \leq\left(1-p_{5}\right) \diamond\left(1-p_{5}\right) \leq\left(1-p_{4}\right) \diamond\left(1-p_{4}\right) \leq 1-p_{0}<p_{2},
\end{aligned}
$$

which is a contradiction. Therefore $S_{(\mu, \nu)}^{I}(\hat{F})$ is a Hausdorff space. The proof for $S_{0(\mu, \nu)}^{I}(\hat{F})$ follows similarly.

Theorem 2.4 $\operatorname{Let} S_{(\mu, \nu)}^{I}(\hat{F})$ be IFNS and $\tau_{(\mu, \nu)}^{I}(\hat{F})$ be a topology on $S_{\mu, \nu)}^{I}(\hat{F})$. A sequence $\left(x_{k}\right) \in$ $S_{(\mu, v)}^{I}(\hat{F})$ converges to $\xi$ iff $\mu\left(\hat{F}_{k}(x)-\xi, t\right) \rightarrow 1$ and $\nu\left(\hat{F}_{k}(x)-\xi, t\right) \rightarrow 0$ as $k \rightarrow \infty$. 
Proof Suppose $x_{k} \rightarrow \xi$, then given $0<r<1$ there exists $k_{0} \in \mathbb{N}$ such that $\left(x_{k}\right) \in B_{x}(r, t)(\hat{F})$ for all $k \geq k_{0}$ given $t>0$. Hence, we have $1-\mu\left(\hat{F}_{k}(x)-\xi, t\right)<r$ and $v\left(\hat{F}_{k}(x)-\xi, t\right)<r$. Therefore $\mu\left(\hat{F}_{k}(x)-\xi, t\right) \rightarrow 1$ and $v\left(\hat{F}_{k}(x)-\xi, t\right) \rightarrow 0$ as $k \rightarrow \infty$.

Conversely, if $\mu\left(\hat{F}_{k}(x)-\xi, t\right) \rightarrow 1$ and $v\left(\hat{F}_{k}(x)-\xi, t\right) \rightarrow 0$ as $k \rightarrow \infty$ holds for each $t>0$. For $0<r<1$, there exists $k_{0} \in \mathbb{N}$ such that $1-\mu\left(\hat{F}_{k}(x)-\xi, t\right)<r$ and $v\left(\hat{F}_{k}(x)-\xi, t\right)<r$ for all $k \geq k_{0}$, which implies $\mu\left(\hat{F}_{k}(x)-\xi, t\right)>1-r$ and $v\left(\hat{F}_{k}(x)-\xi, t\right)<r$. Thus $x_{k} \in B_{x}(r, t)(\hat{F})$ for all $k \geq k_{0}$ and hence $x_{k} \rightarrow \xi$.

\section{Conclusion}

In the present article, we have defined a new kind of sequence spaces $S_{0(\mu, \nu)}^{I}(\hat{F})$ and $S_{(\mu, \nu)}^{I}(\hat{F})$ using Fibonacci difference matrix $\hat{F}$. We studied certain elementary properties and topological properties like linearity, first countability, hausdorfness. These results will give new approach to deal with the problems in science and engineering. The present article is a useful tool to define ideal convergence of generalised Fibonacci difference sequence in intuitionistic fuzzy normed space given by

$$
\begin{aligned}
& c_{0}(\hat{F}(r, s))=\left\{x=\left(x_{k}\right) \in \omega: \lim _{n \rightarrow \infty}\left(r \frac{f_{n}}{f_{n+1}} x_{n}+s \frac{f_{n+1}}{f_{n}} x_{n-1}\right)=0\right\}, \\
& c(\hat{F}(r, s))=\left\{x=\left(x_{k}\right) \in \omega: \lim _{n \rightarrow \infty}\left(r \frac{f_{n}}{f_{n+1}} x_{n}+s \frac{f_{n+1}}{f_{n}} x_{n-1}\right)=l\right\},
\end{aligned}
$$

where $\hat{F}(r, s)=\left\{f_{n k}(r, s)\right\}$ is a double generalised matrix defined as follows:

$$
f_{n k}(r, s)= \begin{cases}s \frac{f_{n+1}}{f_{n}}, & k=n-1, \\ r \frac{f_{n}}{f_{n+1}}, & k=n, \\ 0, & 0 \leq k<n-1 \text { or } k>n\end{cases}
$$

$k, n \in \mathbb{N}$ and $r, s \in \mathbb{R} \backslash\{0\}$. We can study the topological properties of these spaces which will provide a better method to deal with vagueness and inexactness occurring in various fields of science, engineering and economics. Moreover, this theory can be helpful in dealing with problems in population dynamics, quantum particle physics particularly in connections with string and $\epsilon^{\infty}$ theory of El-Naschie.

\section{Acknowledgements}

The authors would like to record their gratitude to the reviewers for their careful reading and making some useful corrections which improved the presentation of the paper.

Funding

This work is financially supported by Nazneen Khan, Assistant Professor in the Department of Mathematics, Taibah University, Medina, Saudi Arabia.

\section{Competing interests}

The authors declare that they have no competing interests.

Authors' contributions

All the authors of this paper have read and agreed to its content and are responsible for all aspects of the accuracy and integrity of the manuscript.

\section{Authors' information}

Vakeel A. Khan received Ph.D degree in Mathematics from Aligarh Muslim University, Aligarh, India. Presently he is an Associate Professor in the Dept. of Mathematics, Aligarh Muslim University. His area of research is Sequence Spaces, and he has published his papers in national and international journals, namely Information Sciences (Elsevier), Applied 
Mathematics Letters (Elsevier), A Journal of Chinese Universities (Springer-Verlag, China). Emrah Evren Kara is an Associate Professor in the Department of Mathematics, Duzce University, Duzce 602002, Turkey. Henna Altaf has done M. Sc. Mathematics from Aligarh Muslim University. Currently she is a research scholar in Aligarh Muslim University. Nazneen Khan is an Assistant Professor in the Department of Mathematics, Taibah University, Medina, Saudi Arabia. Mobeen Ahmad has done M. Sc., M. Phil Mathematics from Aligarh Muslim University. Currently he is a research scholar in Aligarh Muslim University.

\section{Author details}

'Department of Mathematics, Aligarh Muslim University, Aligarh, India. ${ }^{2}$ Department of Mathematics, Duzce University, Duzce, Turkey. ${ }^{3}$ Department of Mathematics, Taibah University, Medina, Saudi Arabia.

\section{Publisher's Note}

Springer Nature remains neutral with regard to jurisdictional claims in published maps and institutional affiliations.

Received: 15 April 2019 Accepted: 9 July 2019 Published online: 18 July 2019

\section{References}

1. Atanassov, K.T.: Intuitionistic fuzzy sets. Fuzzy Sets Syst. 20, 87-96 (1986)

2. Candan, M., Kara, E.E.: A study on topological and geometrical characteristics of new Banach sequence spaces. Gulf J. Math. 3, 67-84 (2015)

3. Das, A., Hazarika, B.: Some properties of generalized Fibonacci difference bounded and $p$-absolutely convergent sequences (2016). Preprint arXiv:1604.00182

4. Das, A., Hazarika, B.: Matrix transformation of Fibonacci band matrix on generalized bv-space and its dual spaces. Bol. Soc. Parana. Mat. 36, 41-52 (2018)

5. Debnath, P.: Lacunary ideal convergence in intuitionistic fuzzy normed linear spaces. Comput. Math. Appl. 63 , 708-715 (2012)

6. Fast, H.: Sur la convergence statistique. Colloq. Math. 2, 241-244 (1951)

7. George, A., Veeramani, P.: On some results in fuzzy metric spaces. Fuzzy Sets Syst. 64, 395-399 (1994)

8. Hazarika, B., Tamanag, K.: On Zweier generalized difference ideal convergent sequences in a locally convex space defined by Musielak-Orlicz function. Bol. Soc. Parana. Mat. 35, 19-37 (2016)

9. Kara, E.E.: Some topological and geometrical properties of new Banach sequence spaces. J. Inequal. Appl. 2013, 38 (2013)

10. Kara, E.E., Ilkhan, M.: On some Banach sequence spaces derived by a new band matrix. Br. J. Math. Comput. Sci. 9, 141-159 (2015)

11. Kara, E.E., Ilkhan, M.: Some properties of generalized Fibonacci sequence spaces. Linear Multilinear Algebra 64, 2208-2223 (2016)

12. Khan, V.A., Esi, A., Fatima, H.: Intuitionistic fuzzy l-convergent double sequence spaces defined by compact operator and modulus function. J. Intell. Fuzzy Syst. 33, 3905-3911 (2017)

13. Khan, V.A., Rababah, R.K.A., Alshlool, K.M.A.S., Abdullah S.A.A., Ahmad, A.: On ideal convergence Fibonacci difference sequence spaces. Adv. Differ. Equ. 2018, 199 (2018)

14. Khan, V.A., Yasmeen, Fatima, H., Altaf, H., Lohani, Q.M.D.: Intuitionistic fuzzy I-convergent sequence spaces defined by compact operator. Cogent Math. 3 (2016)

15. Kostyrko, P., Macaj, M., Šalát, T.: Statistical convergence and I-convergence, Real Anal. Exch. (1999)

16. Michael, A., Erceg, M.A.: Metric spaces in fuzzy set theory. J. Math. Anal. Appl. 69, 205-230 (1979)

17. Mursaleen, M., Lohani, Q.M.D.: Intuitionistic fuzzy 2-normed space and some related concepts. Chaos Solitons Fractals 42, 224-234 (2009)

18. Saadati, R., Park, J.H.: On the intuitionistic fuzzy topological spaces. Chaos Solitons Fractals 27, 331-344 (2006)

19. Šalát, T., Tripathy, B.C., Ziman, M.: On some properties of I-convergence. Tatra Mt. Math. Publ. 28, 274-286 (2004)

20. Šalát, T., Tripathy, B.C., Ziman, M.: On I-convergence field. Ital. J. Pure Appl. Math. 17, 1-8 (2005)

21. Steinhaus, H.: Sur la convergence ordinaire et la convergence asymptotique. Colloq. Math. 2, 73-74 (1951)

22. Tripathy, B., Hazarika, B.: Paranorm /-convergent sequence spaces. Math. Slovaca 59, 485-494 (2009)

23. Tripathy, B.C., Hazarika, B.: Some /-convergent sequence spaces defined by Orlicz functions. Acta Math. Appl. Sin. Engl. Ser. 27, 149-154 (2011)

24. Zadeh, L.A.: Fuzzy sets. Inf. Control 8, 338-353 (1965) 